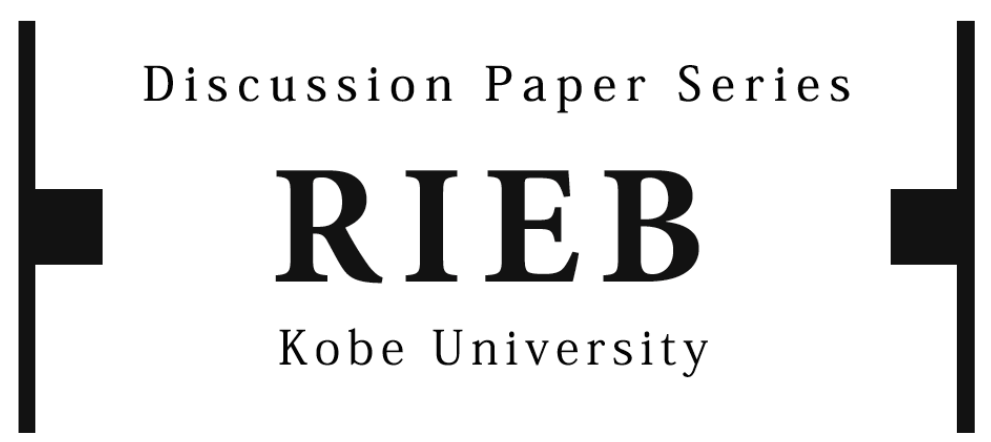

DP2015-42

Regime-Switching Sunspot Equilibria in a One-Sector Growth Model with Aggregate Decreasing Returns and Small Externalities

Takashi KAMIHIGASHI

December 1, 2015

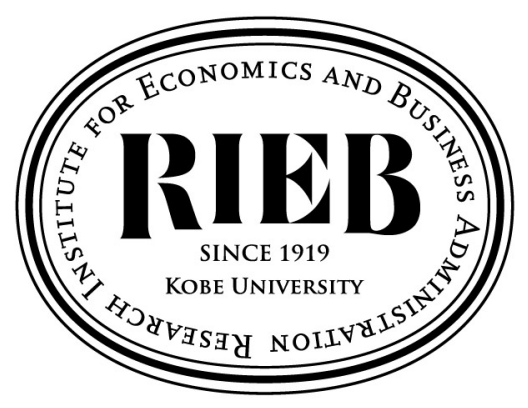

Research Institute for Economics and Business Administration Kobe University 


\title{
Regime-Switching Sunspot Equilibria in a One-Sector Growth Model with Aggregate Decreasing Returns and Small Externalities
}

\author{
Takashi Kamihigashi*
}

December 1, 2015

\begin{abstract}
This paper shows that regime-switching sunspot equilibria easily arise in a one-sector growth model with aggregate decreasing returns and arbitrarily small externalities. We construct a regime-switching sunspot equilibrium in the case where the utility function of consumption is linear. We also construct a stochastic optimal growth model whose optimal process is a regime-switching sunspot equilibrium of the original economy in the case where there is no capital externality. We illustrate our results with numerical examples.
\end{abstract}

\footnotetext{
*RIEB, Kobe University, Rokkodai, Nada, Kobe 657-8501 Japan
}

Email: tkamihig@rieb.kobe-u.ac.jp 


\section{Introduction}

In macroeconomics, sunspot equilibria are often associated with local indeterminacy, or the existence of a locally stable steady state. In the context of growth models, the phenomenon of local indeterminacy has been well known since Benhabib and Farmer (1994) and Farmer and Guo (1994). While earlier results required excessively large degrees of increasing returns and externalities 11 local indeterminacy has been established for various settings under less objectionable assumptions, including decreasing returns to labor (e.g., Pelloni and Waldmann, 1998), moderate externalities (Dufourt et al., 2015), aggregate constant returns to scale (e.g., Benhabib et al., 2000; Mino, 2001), and arbitrarily small increasing returns and externalities (e.g, Kamihigashi, 2002; Pintus, 2006).

In this paper we point out the possibility out an alternative mechanism that gives rise to sunspot equilibria in an economy with aggregate decreasing returns and arbitrarily small externalities. In particular, instead of small fluctuations around a locally indeterminate steady state, we consider large fluctuations caused by a regime-switching sunspot process, which is assumed to be a two-state Markov chain. Labor supply is positive in one state, while it is zero in the other. Although the regime-switching sunspot equilibria that we construct are rather extreme, it can easily be seen that this type of sunspot equilibrium is widespread in models with aggregate externalities.

We present two main results. First, assuming that the utility function of consumption is linear, we construct a regime-switching sunspot equilibrium by recursively solving the Euler condition for capital and the first-order condition for labor supply and by verifying the transversality condition. This approach is possible when utility of consumption is linear, in which case the three conditions mentioned above do not depend on consumption. Second, assuming that there is no capital externality, we establish the existence of a regime-switching sunspot equilibrium by constructing a stochastic optimal growth model whose optimal process is a sunspot equilibrium of the original economy. This is somewhat similar to the observational equivalence result shown by Kamihigashi (1996); in contrast to the latter result, however, here we only use the Euler condition for capital, the first-order condition for labor supply, and the transversality condition to verify that an optimal process for

\footnotetext{
${ }^{1}$ See Benhabib and Farmer (1999) for a survey of earlier results. See Grandmont (1989, 1991) for discussion of relations between local stability properties of a steady state and the possibility of sunspot equilibria.
} 
the stochastic optimal growth model can be seen as a sunspot equilibrium. Both results are illustrated with numerical examples.

In addition to the results on local indeterminacy mentioned above, other related results include those on global indeterminacy (e.g., Drugeon and Venditti, 2001; Coury and Wen, 2009) and regime-switching sunspot equilibria (e.g., Drugeon and Wigniolle, 1996) ${ }^{2}$ Our results differ in that we consider a standard one-sector growth model with aggregate decreasing returns and small externalities ${ }^{3}$ Indeed, our model is a variant of the Farmer-Guo (1994) model with aggregate decreasing returns and small exteralities.

The rest of the paper is organized as follows. In the next section we present the model along with basic definitions and assumptions. In Section 3 we show a standard result that offers a sufficient set of conditions for a feasible process to be an equilibrium. In Section 4 we present our mains results along with numerical examples. In Section 5 we conclude the paper by discussing possible extensions. We provide longer proofs in two appendices.

\section{The Model}

We consider an economy with many agents, each of whom solves the following maximization problem:

$$
\begin{array}{cl}
\max _{\left\{c_{t}, n_{t}, k_{t+1}\right\}_{t=0}^{\infty}} E \sum_{t=0}^{\infty} \beta^{t}\left[u\left(c_{t}\right)-w\left(n_{t}\right)\right] \\
\text { s.t. } \forall t \in \mathbb{Z}_{+}, \quad c_{t}+k_{t+1}=f\left(k_{t}, n_{t}, K_{t}, N_{t}\right)+(1-\delta) k_{t}, \\
c_{t}, k_{t+1} \geqslant 0, \quad n_{t} \in[0,1],
\end{array}
$$

where $c_{t}$ is consumption, $n_{t}$ is labor supply, $k_{t}$ is the capital stock at the beginning of period $t, N_{t}$ is aggregate labor supply, and $K_{t}$ is the aggregate capital stock. The utility function $u$ of consumption, the disutility function $w$ of labor supply, and the production function $f$ are specified below. The discount factor $\beta$ and the depreciation rate $\delta$ satisfy

$$
\beta, \delta \in(0,1)
$$

\footnotetext{
${ }^{2}$ See Clain-Chamosset-Yvrard and Kamihigashi (2015) for regime-switching sunspot equilibria in a two-country model with asset bubbles.

${ }^{3}$ It is shown in Kamihigashi (2015) that multiple steady states are possible eve without externalities.
} 
In the above maximization problem, the initial capital stock $k_{0}>0$ and the stochastic processes $\left\{K_{t}\right\}_{t=0}^{\infty}$ and $\left\{N_{t}\right\}_{t=0}^{\infty}$ are taken as given. However, in equilibrium, we have

$$
\forall t \in \mathbb{Z}_{+}, \quad K_{t}=k_{t}, \quad N_{t}=n_{t} .
$$

To formally define an equilibrium of this economy, we first define a preequilibrium as a five-dimensional stochastic process $\left\{c_{t}, n_{t}, k_{t}, N_{t}, K_{t}\right\}_{t=0}^{\infty}$ such that $\left\{c_{t}, n_{t}, k_{t+1}\right\}_{t=0}^{\infty}$ solves the maximization problem 2.1)-2.3) given $k_{0}>0$ and the nonnegative processes $\left\{N_{t}\right\}_{t=0}^{\infty}$ and $\left\{K_{t}\right\}_{t=0}^{\infty}$. We define an equilibrium as a three-dimensional stochastic process $\left\{c_{t}, n_{t}, k_{t}\right\}_{t=0}^{\infty}$ such that the five-dimensional stochastic process $\left\{c_{t}, n_{t}, k_{t}, n_{t}, k_{t}\right\}_{t=0}^{\infty}$ is a pre-equilibrium. We also define a feasible process as a three-dimensional stochastic process $\left\{c_{t}, n_{t}, k_{t}\right\}_{t=0}^{\infty}$ satisfying (2.2), 2.3), and 2.5).

We specify the functions $u, w$, and $f$ as follows:

$$
\begin{aligned}
u(c) & =\frac{c^{1-\sigma}-1}{1-\sigma}, \\
w(n) & =\eta \frac{n^{\gamma+1}}{\gamma+1}, \\
f(k, n, K, N) & =\theta k^{\alpha} n^{\rho} K^{\bar{\alpha}} N^{\bar{\rho}} .
\end{aligned}
$$

We impose the following restrictions on the parameters:

$$
\begin{gathered}
\sigma \in[0,1], \\
\theta, \alpha, \rho, \eta>0, \\
\bar{\alpha}, \bar{\rho}, \gamma \geqslant 0, \\
\bar{\alpha}+\alpha+\bar{\rho}+\rho \leqslant 1 .
\end{gathered}
$$

If $\sigma=1$, then it is understood that $u(c)=\ln c$. Since $\sigma \in[0,1]$ by $(2.9)$, $u$ is bounded below unless $\sigma=1$. The inequality in 2.12 means that the production function exhibits decreasing returns to scale at the aggregate level. In what follows, we use the nonparametric forms $u, w$, and $f$ and the parametric forms given by (2.6)-(2.8) above interchangeably.

Let $\widehat{k}$ be the unique strictly positive capital stock $k>0$ such that $\theta k^{\alpha+\bar{\alpha}}=$ $\delta k$. The capital stock $\hat{k}$ is the maximum sustainable capital stock. It has the property that for any feasible process $\left\{c_{t}, n_{t}, k_{t}\right\}_{t=0}^{\infty}$ we have

$$
\forall k_{t} \in \mathbb{Z}_{+}, \quad k_{t} \leqslant \max \left\{k_{0}, \hat{k}\right\} .
$$

Therefore all equilibria are bounded. 


\section{Sufficient Optimality Conditions}

It follows from $(2.8)$ that

$$
\forall k, n>0, \quad f_{1}(k, n, k, 0)=f_{2}(k, n, k, 0)=0,
$$

where $f_{i}(\cdot, \cdot, \cdot, \cdot)$ is the derivative of $f$ with respect to the $i$ th argument. To simplify notation, for $i=1,2$, we define

$$
f_{i}(k, n)=f_{i}(k, n, k, n) .
$$

For $k, n \geqslant 0$ we also define

$$
\zeta=1-\delta, \quad g(k, n)=f(k, n, k, n)+\zeta k .
$$

The first-order condition for labor supply $n_{t}$ in period $t$ is given by

$$
u^{\prime}\left(c_{t}\right) f_{2}\left(k_{t}, n_{t}\right)-w^{\prime}\left(n_{t}\right) \begin{cases}=0 & \text { if } n_{t} \in(0,1) \\ \geqslant 0 & \text { if } n_{t}=1 \\ \leqslant 0 & \text { if } n_{t}=0\end{cases}
$$

Note from (3.1) that $n_{t}=0$ is always a solution to (3.4). This observation is the basis for our construction of sunspot equilibria. On the other hand, as long as $N_{t}>0$ and $k_{t}, K_{t}>0$, it is always optimal to choose strictly positive labor supply since $f_{2}(k, 0, k, N)=\infty$ for any $k, N>0$.

The stochastic Euler condition for the capital stock $k_{t+1}$ at the beginning of period $t+1$ can be written as

$$
\begin{aligned}
& -u^{\prime}\left(c_{t}\right)+\beta E_{t} u^{\prime}\left(c_{t+1}\right)\left[f_{1}\left(k_{t+1}, n_{t+1}\right)+\zeta\right] \\
& \begin{cases}=0 & \text { if } k_{t+1} \in\left(0, g\left(k_{t}, n_{t}\right)\right), \\
\geqslant & \text { if } k_{t+1}=g\left(k_{t}, n_{t}\right), \\
\leqslant & \text { if } k_{t+1}=0 .\end{cases}
\end{aligned}
$$

We need to consider corner solutions since one of the results shown in the next section assumes that the utility function of consumption is linear. However, as long as labor supply is strictly positive with strictly positive probability in period $t+1$, there is a solution to 3.5 with $k_{t+1}>0$, because $\lim _{k \downarrow 0} f_{1}(k, n)=\infty$ for any $n>0$. 
The transversality condition is

$$
\lim _{T \rightarrow \infty} \beta^{T} E u^{\prime}\left(c_{T}\right) k_{T+1}=0 .
$$

See Kamihigashi (2003, 2005) for more on transversality conditions for stochastic problems.

The following result shows that the above first-order conditions along with the transversality condition are sufficient for a feasible process to be an equilibrium. The proof is a stochastic version of the standard sufficiency proof.

Lemma 3.1. A feasible process $\left\{c_{t}, n_{t}, k_{t}\right\}_{t=0}^{\infty}$ is an equilibrium if it satisfies (3.4) and (3.5) for all $t \in \mathbb{Z}_{+}$and (3.6).

Proof. See Appendix A.

\section{Sunspot Equilibria}

\subsection{Common Structure}

In this paper we consider a special type of sunspot equilibrium by taking a regime-switching sunspot process $\left\{s_{t}\right\}$ as given. In particular, we assume that there are two sunspot states, 0 and 1 , and $\left\{s_{t}\right\}$ is a two-state Markov chain with transition matrix

$$
\left[\begin{array}{ll}
p_{00} & p_{01} \\
p_{10} & p_{11}
\end{array}\right]
$$

where $p_{i j}$ is the probability that $s_{t+1}=j$ given $s_{t}=i$ for $i, j \in\{0,1\}$. To simplify the analysis, we assume that $p_{i j}>0$ for all $i, j \in\{0,1\}$. Since (4.1) is a transition Matrix, we have

$$
p_{00}+p_{01}=p_{10}+p_{11}=1 .
$$

In what follows, all stochastic processes (sequences) are assumed to be adapted to the $\sigma$-field generated by the Markov chain $\left\{s_{t}\right\}_{t=0}^{\infty}$. This simply means that any variable indexed by $t$ is a function of the history of sunspot states $s_{0}, s_{1}, \ldots, s_{t}$ up to period $t$. Since $\left\{s_{t}\right\}$ is a sunspot process, it does not directly affect the fundamentals of the economy. An equilibrium $\left\{c_{t}, n_{t}, k_{t}\right\}$ is a 
sunspot equilibrium if it depends on the sunspot process $\left\{s_{t}\right\}$ in a nontrivial way.

To see the possibility of a sunspot equilibrium, suppose that in the maximization problem (2.1)-(2.3), we have

$$
N_{t} \begin{cases}>0 & \text { if } s_{t}=1, \\ =0 & \text { if } s_{t}=0 .\end{cases}
$$

Then provided that $k_{t}=K_{t}>0$ for all $t \in \mathbb{Z}_{+}$, we must have

$$
n_{t} \begin{cases}>0 & \text { if } s_{t}=1 \\ =0 & \text { if } s_{t}=0\end{cases}
$$

For the rest of the paper, we assume that $k_{t}=K_{t}>0$ for all $t \in \mathbb{Z}_{+}$, focusing on regime-switching sunspot equilibria satisfying (4.3) and (4.4).

Under (4.3) the first-order condition (3.4) for $n_{t}$ can be written as

$$
\begin{aligned}
& s_{t}=1 \quad \Rightarrow \quad u^{\prime}\left(c_{t}\right) f_{2}\left(k_{t}, n_{t}\right)-w^{\prime}\left(n_{t}\right) \begin{cases}=0 & \text { if } n_{t} \in(0,1) \\
0 & \text { if } n_{t}=1\end{cases} \\
& s_{t}=0 \quad \Rightarrow \quad n_{t}=0 .
\end{aligned}
$$

In (4.5) we do not need to consider the case $n_{t}=0$ since $f_{2}(k, 0, k, N)=\infty$ for any $k, N>0$ by (2.8), (2.10), and (2.12), as mentioned above. On the other hand, if $s_{t}=0$, then $N_{t}=0$ by 4.3$)$; thus $n_{t}=0$ since $f_{2}(k, n, k, 0)=0$ for any $k>0$ and $n \geqslant 0$.

\subsection{Linear Utility of Consumption}

One way to show the existence of a sunspot equilibrium is by using Lemma 3.1 to explicitly construct a sunspot equilibrium $\left\{c_{t}, n_{t}, k_{t}\right\}$ satisfying (4.4). To do so, we need to verify the Euler condition (3.5) and the transversality condition (3.6) in addition to (4.5) and 4.6). Although this is not easy to do in general, we can explicitly construct a sunspot equilibrium using these conditions if we assume that the utility function of consumption is linear, in which case none of the conditions depends on consumption (except for feasibility). We consider this special case in the following result.

Proposition 4.1. Suppose that $\sigma=0$. Then there exists a sunspot equilibrium satisfying (4.4). 
Proof. See Appendix B.

The sunspot equilibrium constructed in the proof of Proposition 4.1 is generated by the following system of equations:

$$
\begin{aligned}
n_{t} & =m\left(k_{t}, s_{t}\right), \\
k_{t+1} & =\min \left\{q\left(p_{s_{t} 1}\right), g\left(k_{t}, n_{t}\right)\right\}, \\
c_{t} & =g\left(k_{t}, n_{t}\right)-k_{t+1},
\end{aligned}
$$

where $m(\cdot, \cdot)$ and $q(\cdot)$ are given by $(\mathrm{B} .5)$ and $(\mathrm{B} .14)$, respectively, in Appendix $\mathrm{B}$, and $p_{s_{t} 1}$ is equal to $p_{01}$ or $p_{11}$ depending on $s_{t}$ is equal to 0 or 1 . Given $k_{t}>0$ and $s_{t} \in\{0,1\}, n_{t}$ is determined by 4.7$), k_{t+1}$ is determined by (4.8), and $c_{t}$ is determined by (4.9). With a new sunspot variable $s_{t+1}$ drawn according to (4.1), $n_{t+1}$ is determined by (4.7) again, and so one.

Figure 1 depicts the functions in 4.7$)-(4.9)$ with the following parameter values:

$$
\begin{gathered}
\beta=0.9, \quad \eta=1, \quad \gamma=0.1, \quad p_{01}=0.2, \quad p_{11}=0.8 \\
\delta=0.05, \quad \theta=3, \quad \rho=0.55, \quad \bar{\rho}=0.03 \\
\sigma=0, \quad \alpha=0.35, \quad \bar{\alpha}=0.02 .
\end{gathered}
$$

Figure 2 shows sample paths for sunspot states, capital, labor, and consumption generated by (4.7)-(4.9). The sample path for labor supply $n_{t}$ closely follows the pattern of sunspot states $s_{t}$, as expected from (4.4). The sample paths for capital and consumption inherit the same pattern to a large extent.

There is also a feature specific to consumption, which rises to its highest level when the sunspot state changes from 1 to 0 after maintaining the state of 1 for a few periods. This is expected from the consumption function in Figure 1. Note that this function is increasing in $k_{t}$ but, unlike the labor and capital functions, decreasing in $s_{t}$ in the sense that consumption is higher when $s_{t}=0$ than when $s_{t}=1$.

\subsection{Stochastic Optimal Growth}

One can conjecture that the feature of the consumption function mentioned above is not due to the presence of externalities; rather it may be a conse- 

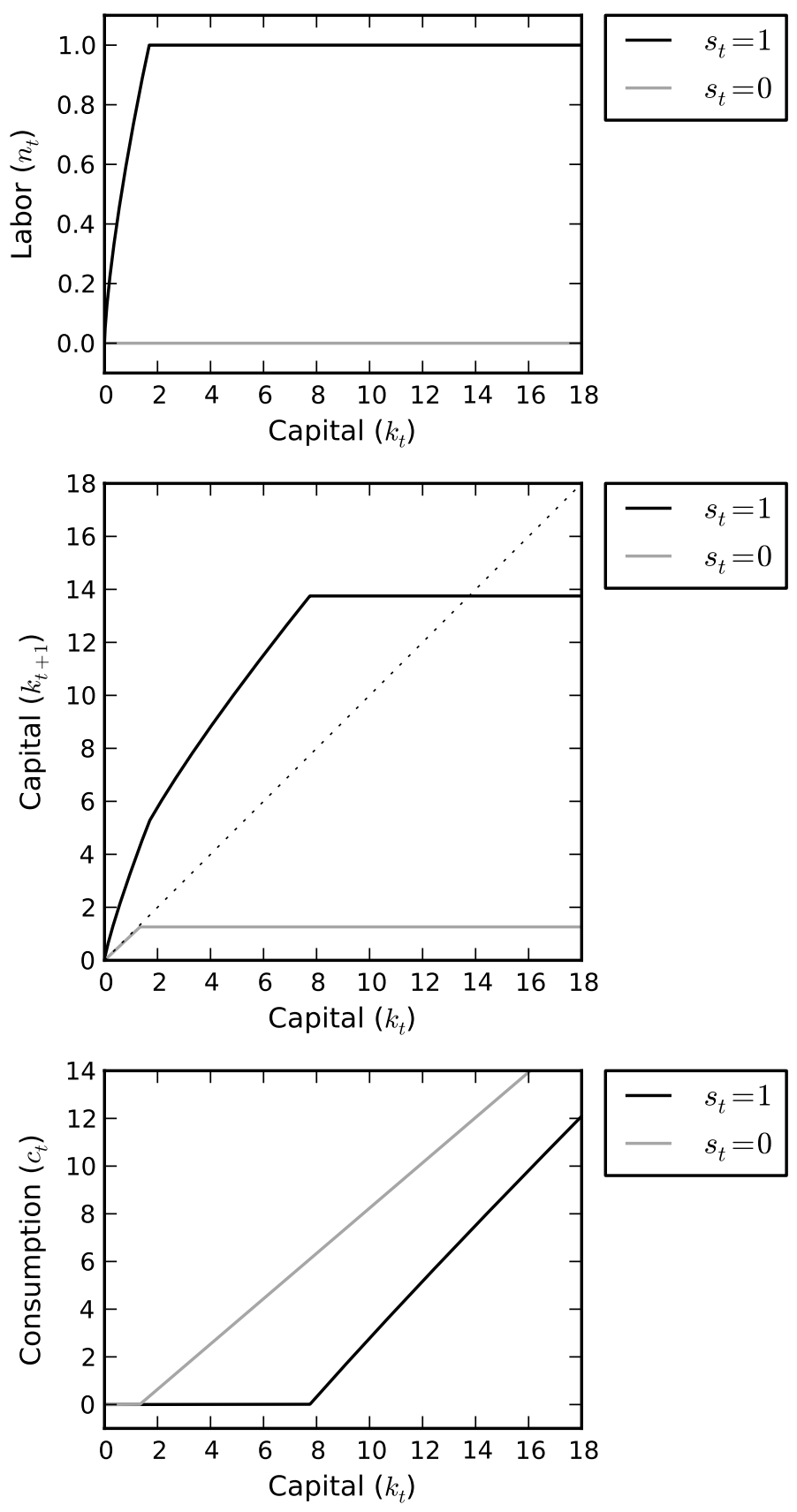

Figure 1: Regime-switching sunspot equilibria under 4.7 - 4.9) with parameter values given by $4.10-4.12$ 
Sunspot $\left(s_{t}, t=0, \ldots, 100\right)$
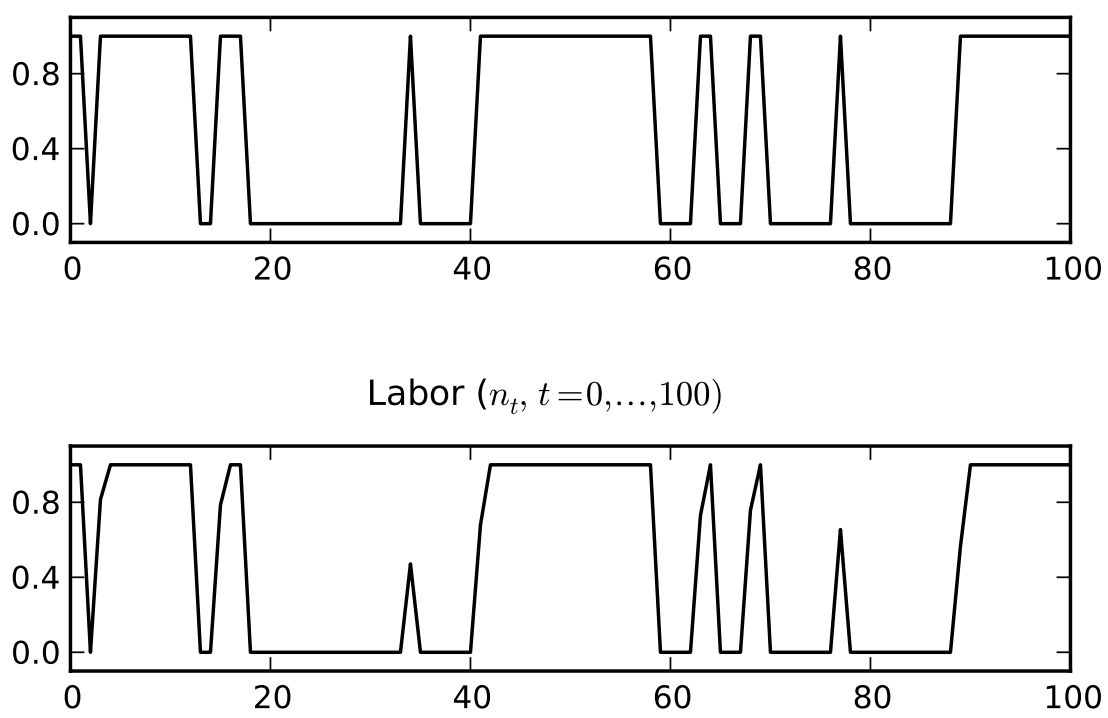

Capital $\left(k_{t}, t=0, \ldots, 100\right)$

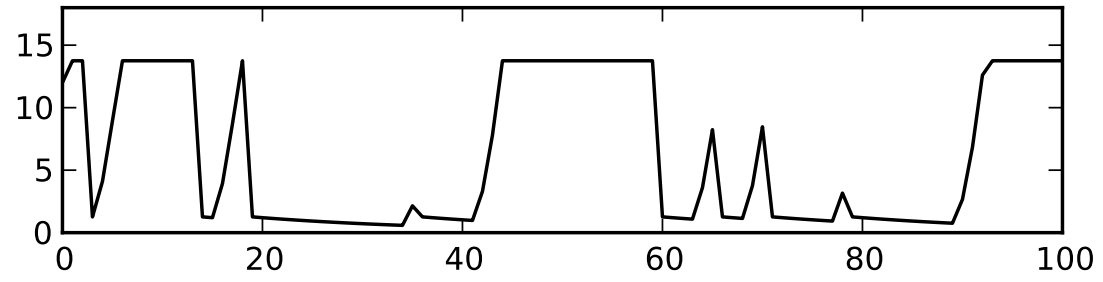

Consumption $\left(c_{t}, t=0, \ldots, 100\right)$

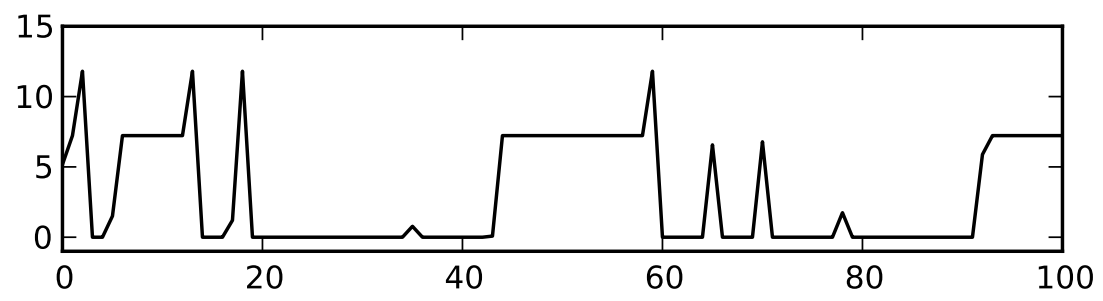

Figure 2: Sample paths for sunspot states, capital, labor, and consumption generated by (4.7)- 4.9) with parameter values given by 4.10-(4.12). 
quence of optimal behavior. To examine this possibility, consider the following stochastic optimal growth model:

$$
\begin{array}{cl}
\max _{\left\{c_{t}, n_{t}, k_{t+1}\right\}_{t=0}^{\infty}} & E \sum_{t=0}^{\infty} \beta^{t}\left[u\left(c_{t}\right)-w\left(n_{t}\right)\right] \\
\text { s.t. } \forall t \in \mathbb{Z}_{+}, & c_{t}+k_{t+1}=s_{t}\left(k_{t}\right)^{\alpha+\bar{\alpha}}\left(n_{t}\right)^{\rho+\bar{\rho}}+(1-\delta) k_{t}, \\
& c_{t}, k_{t+1} \geqslant 0, \quad n_{t} \in[0,1],
\end{array}
$$

where $\left\{s_{t}\right\}$ is the same two-state Markov process following 4.1). This problem has the same aggregate production function, but the externalities are internalized whenever $s_{t}=1$. Since output is zero whenever $s_{t}=0$, the problem inherits the pattern of (4.3).

Figure 3 depicts the optimal policy functions for the stochastic optimal growth model 4.13)-4.15) under 4.10-4.12). These functions are given by $4.7-4.9$ with

$$
\alpha=0.37, \quad \rho=0.58, \quad \bar{\alpha}=\bar{\rho}=0 .
$$

Note that the consumption function in Figure 3 is decreasing in $s_{t}$ like that in Figure 1; in fact, consumption with $s_{t}=1$ is even lower than in Figure 1. This is because, as can be seen from the capital function in Figure 3, more capital is accumulated in the stochastic optimal growth model 4.13$)-(4.15)$ than in the original economy with externalities under 4.10 4.12). The functions in Figure 3 constitute an example of a stochastic optimal growth model in which consumption is decreasing in correlated productivity, while capital and labor are increasing in productivity. This is in sharp contrast to the Brock-Mirman (1972) model with i.i.d. productivity shocks, where consumption is always increasing in productivity; see Kamihigashi (2008, Theorem 2.1).

Figure 4 shows sample paths generated by these optimal policy functions with the same sunspot states as in Figure 2. These paths are similar to those in Figure 2, but the capital path is overall higher, and the peaks of consumption are also higher than in Figure 2 .

\subsection{No Capital Externality}

Our analysis in the previous two subsections suggests that there is a close connection between the sunspot equilibria of the original economy and the 

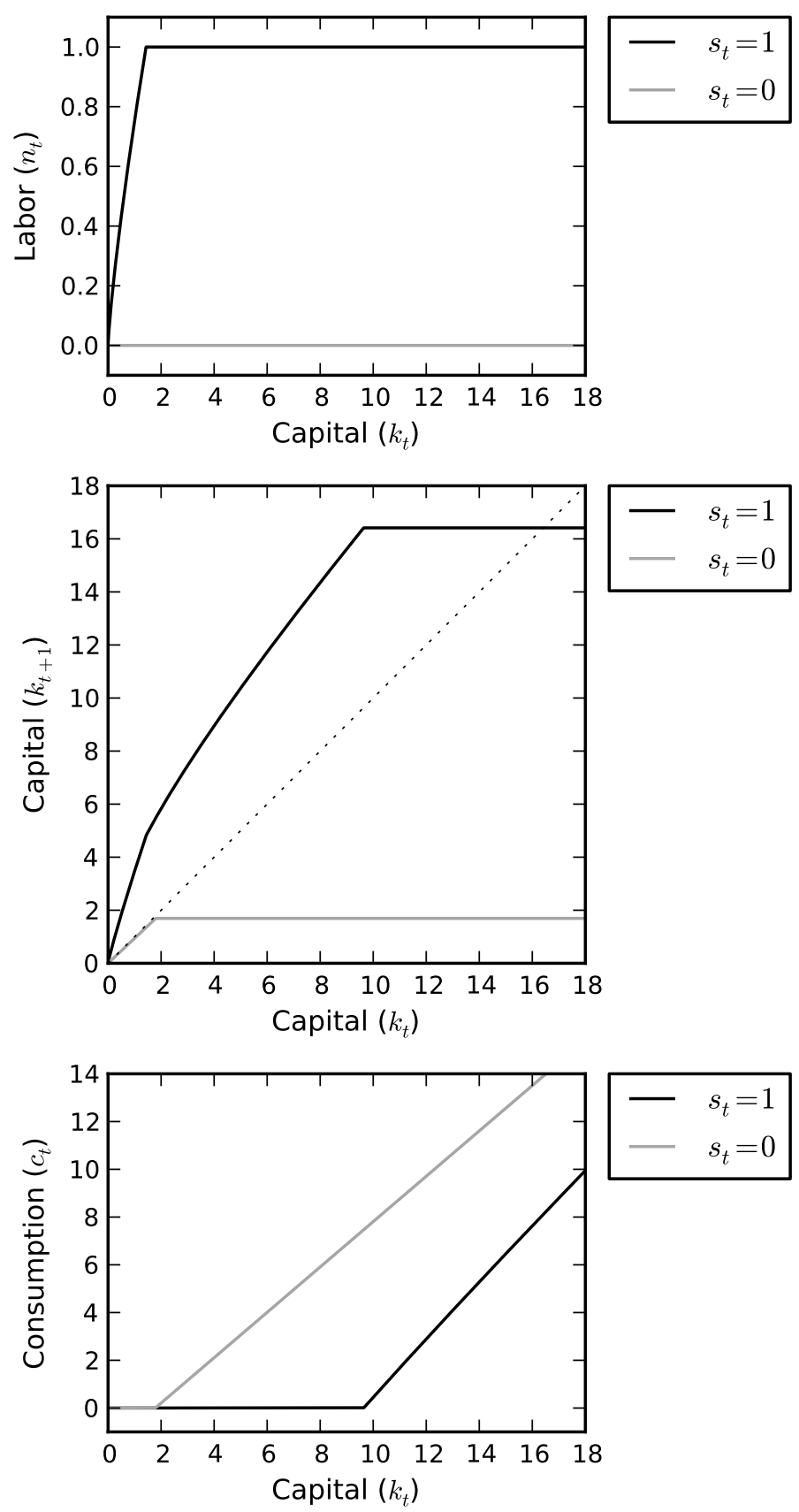

Figure 3: Optimal policy functions for 4.13 4.15 
Sunspot $\left(s_{t}, t=0, \ldots, 100\right)$

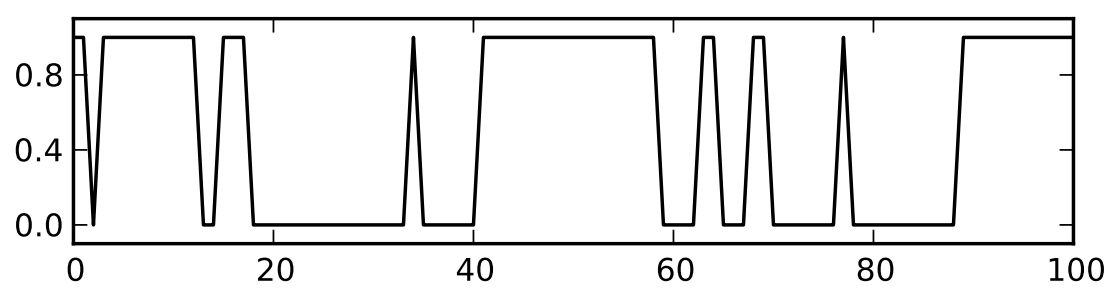

Labor $\left(n_{t}, t=0, \ldots, 100\right)$

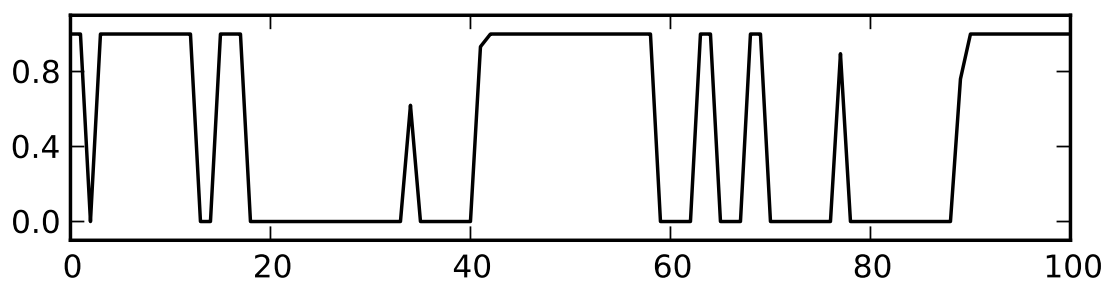

Capital $\left(k_{t}, t=0, \ldots, 100\right)$

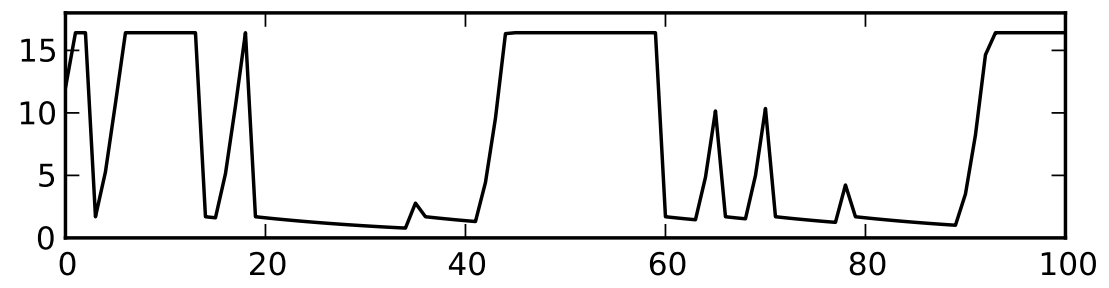

Consumption $\left(c_{t}, t=0, \ldots, 100\right)$

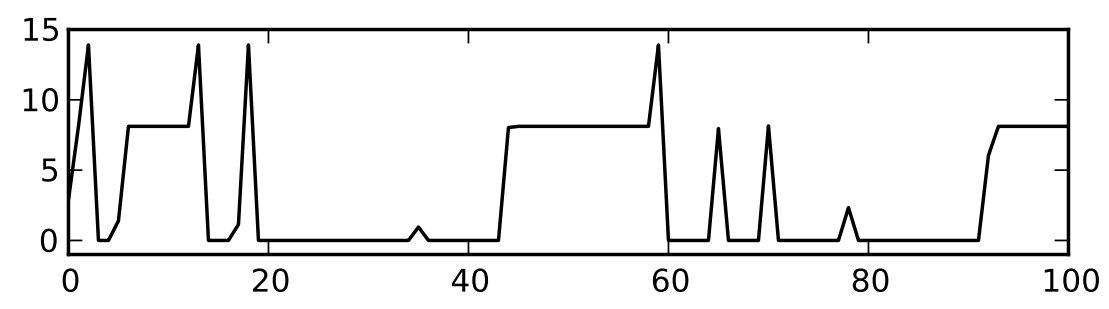

Figure 4: Sample paths for sunspot states, capital, labor, and consumption 
optimal process of some stochastic optimal growth model. Although this is not trivial to show in general, it is fairly easy to establish a clear connection in the absence of capital externalities. The proof of the following result utilizes this observation.

Proposition 4.2. Suppose that $\bar{\alpha}=0$. Then there exists a sunspot equilibrium satisfying (4.4).

Proof. See Appendix C

The condition $\bar{\alpha}=0$ in the above proposition means that there is no capital externality. In the proof of Proposition 4.2, we consider the following stochastic optimal growth model:

$$
\begin{gathered}
\max _{\left\{c_{t}, n_{t}, k_{t+1}\right\}_{t=0}^{\infty}} E \sum_{t=0}^{\infty} \beta^{t}\left[u\left(c_{t}\right)-\frac{\rho+\bar{\rho}}{\rho} w\left(n_{t}\right)\right] \\
\text { s.t. } \forall t \geqslant 0, \quad c_{t}+k_{t+1}=s_{t} \theta\left(k_{t}\right)^{\alpha}\left(n_{t}\right)^{\rho+\bar{\rho}}+\zeta k_{t}, \\
c_{t}, k_{t+1} \geqslant 0, \quad n_{t} \in[0,1],
\end{gathered}
$$

where $\left\{s_{t}\right\}_{t=0}^{\infty}$ is the same two-state Markov chain following (4.1). In the proof, we show that the Euler condition for $k_{t+1}$, the first order condition for $n_{t}$, and the transversality condition for the above problelm are necessary for optimality, and equivalent to the sufficient optimality conditions for the original economy $(2.1)-(2.3)$ with $\bar{\alpha}=0$. We can thus establish the existence of a sunspot equilibrium by showing the existence of an optimal process for the above stochastic optimal growth model.

The Bellman equation for $4.17-(4.19$ can be written as

$$
\begin{aligned}
v\left(k_{t}\right)=\max _{c_{t}, n_{t}, k_{t+1}}\left\{u\left(c_{t}\right)-\frac{\rho+\bar{\rho}}{\rho} w\left(n_{t}\right)+\beta E_{t} v\left(k_{t+1}\right)\right\} \\
\text { s.t. } \quad c_{t}+k_{t+1}=s_{t} \theta\left(k_{t}\right)^{\alpha}\left(n_{t}\right)^{\rho+\bar{\rho}}+\zeta k_{t}, \\
c_{t}, k_{t+1} \geqslant 0, \quad n_{t} \in[0,1] .
\end{aligned}
$$

By the proof of Proposition 4.2, the optimal policy functions for the above Bellman equation can be interpreted as a regime-switching sunspot equilibrium. We assume the same parameter values as in (4.10) and (4.11), but we replace the values of $\sigma, \alpha$, and $\bar{\alpha}$ as follows:

$$
\sigma=0.01, \quad \alpha=0.37, \quad \bar{\alpha}=0 .
$$


With the above values of $\alpha$ and $\bar{\alpha}$, the aggregate production function $f(k, n, k, n)$ remains the same as under (4.11).

Figure 5 shows the optimal policy functions for the Bellman equation (4.20)-(4.22) under the above parameter values. These functions are computed by numerically solving the Bellman equation using modified policy iteration (e.g., Puterman, 2005) with 5,000 equally spaced grid points.

Figure 6 shows sample paths for sunspot states, labor, capital, and consumption generated by the functions in Figure 5. Compared to those in Figure 2, the sample paths in Figure 6 appear less extreme. Capital accumulates while the sunspot state is 1 , and decumulates while it is 0 . Consumption follows almost exactly the same pattern, while labor supply moves in the opposite directions, as expected from the labor and consumption functions in Figure 5 .

\section{Concluding Comments}

In this paper we showed that regime-switching sunspot equilibria easily arise in a one-sector growth model with aggregate decreasing returns and small externalities. We explicitly constructed a regime-switching sunspot equilibrium in the case where the utility function of consumption is linear. We also constructed a stochastic optimal growth model whose optimal process is a regime-switching sunspot equilibrium of the original economy in the case where there is no capital externality. Although we assumed aggregate decreasing returns to scale throughout the paper, one can easily see that our analysis can be extended to models with increasing returns and large externalities. In concluding the paper, we discuss some possible ways to extend our analysis.

First, although we only considered rather extreme sunspot equilibria such that labor supply and output are zero when the sunspot state is zero, more realistic equilibria can be constructed based on a similar approach. For example, consider a one-sector growth model externalities in which the first-order condition for labor supply has multiple solutions. One can easily construct such a model if one is allowed to assume a general form of externalities. In such a model, one can construct a sunspot equilibrium that switches between the mutiple solutions of the first-order condition for labor supply depending on the sunspot state.

Second, though we focused on sunspot equilibria, it is also possible to 

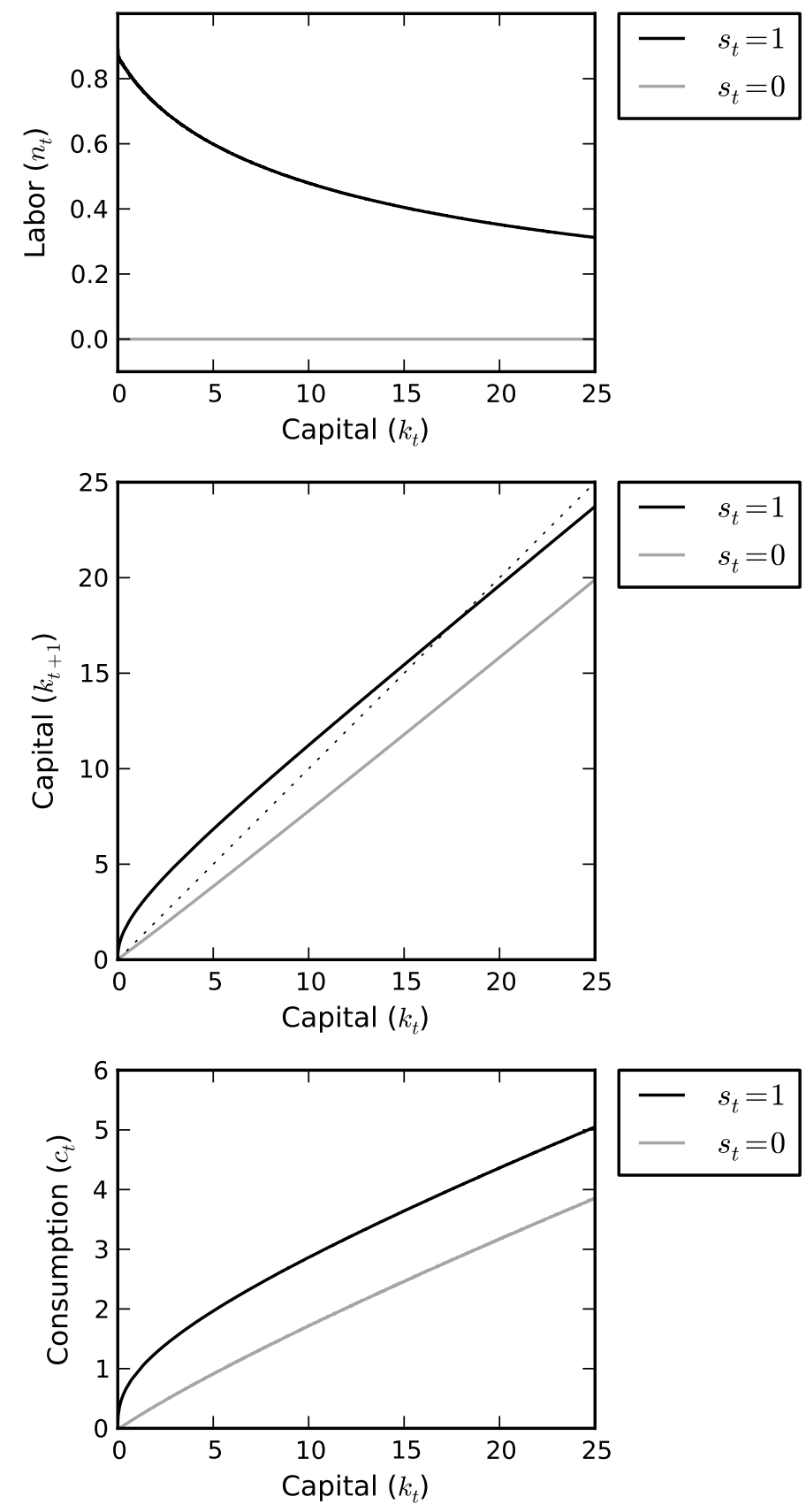

Figure 5: Optimal policy functions for 4.20- 4.22 and regime-switching sunspot equilibria for (2.1)-2.3 under 4.10, 4.11), and 4.23 

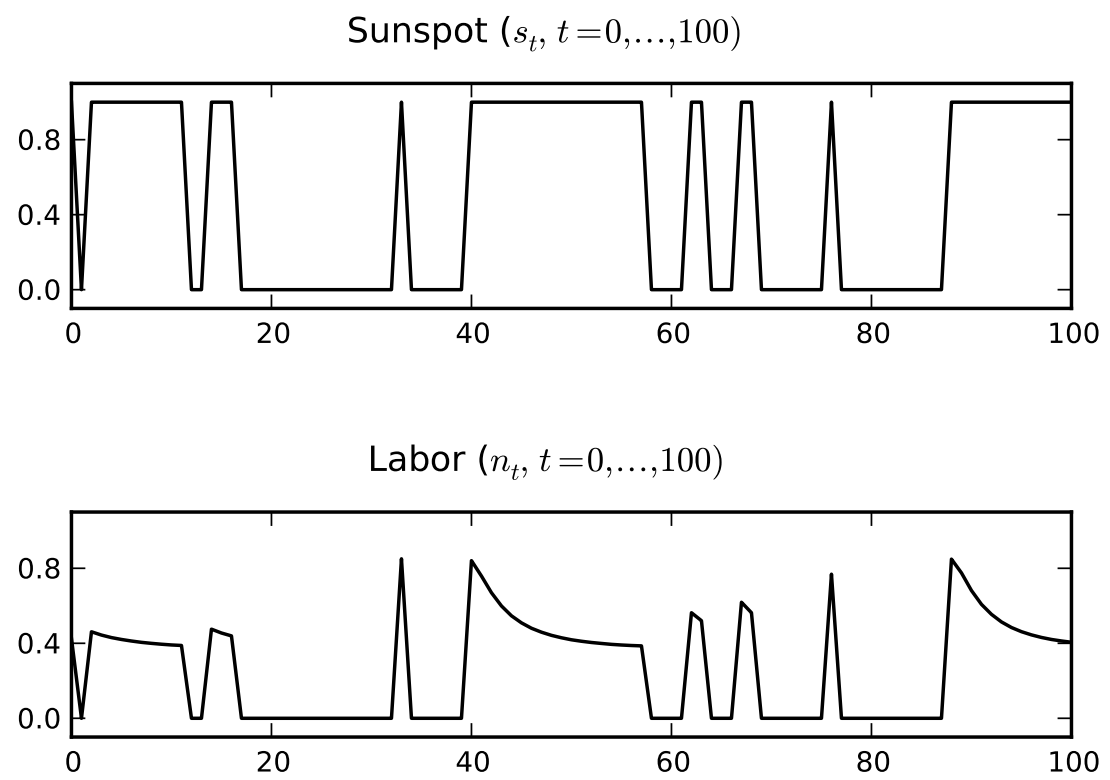

Capital $\left(k_{t}, t=0, \ldots, 100\right)$

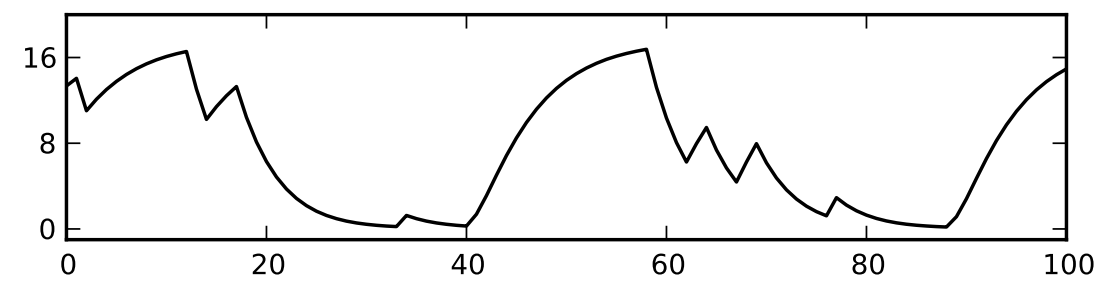

Consumption $\left(c_{t}, t=0, \ldots, 100\right)$

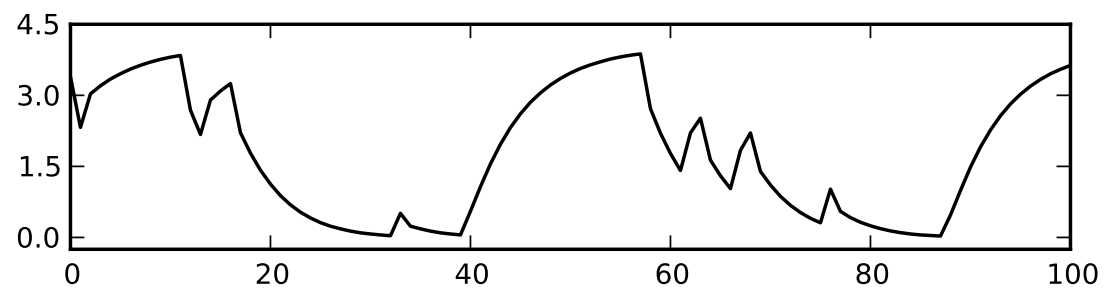

Figure 6: Sample paths for sunspot states, capital, labor, and consumption under 4.10, 4.11, and 4.23 
construct deterministic equilibria that exhibit chaotic dynamics. For example, one can take a deterministic sequence of states $s_{t}$ each of which is either 0 or 1 , and solve the deterministic version of the maximization problem 4.17)-4.19). Then the resulting optimal path follows the pattern of the sequence $\left\{s_{t}\right\}$. This can be thought of as an example of symbolic dynamics; see Kamihigashi (1999) for economic applications of symbolic dynamics.

Finally, in Proposition 4.2 we only considered the case in which there is no capital externality. Although it seems impossible to extend the same approach to models with capital externalities (as long as the capital depreciation rate is less than one), there is a way to deal with such models. In particular, if one allows for nonlinear discounting along the lines of Kamihigashi (2002), it is possible to construct a stochastic optimal growth model whose optimal process is a sunspot equilibrium of the original economy.

\section{Appendix A Proof of Lemma 3.1}

Let $\left\{c_{t}^{*}, n_{t}^{*}, k_{t}^{*}\right\}_{t=0}^{\infty}$ be a feasible process satisfying $\left.3.4-3.6\right)$ (with $c_{t}^{*}, n_{t}^{*}, k_{t}^{*}$ replacing $\left.c_{t}, n_{t}, k_{t}\right)$. To simplify notation, for $t \in \mathbb{Z}_{+}$and $i=1,2$ we define

$$
f(t)=f\left(k_{t}^{*}, n_{t}^{*}, k_{t}^{*}, n_{t}^{*}\right)
$$

We are to show that for any feasible process $\left\{c_{t}, n_{t}, k_{t}\right\}_{t=0}^{\infty}$, we have

$$
E \sum_{t=0}^{\infty} \beta^{t}\left[u\left(c_{t}\right)-w\left(n_{t}\right)\right]-E \sum_{t=0}^{\infty} \beta^{t}\left[u\left(c_{t}^{*}\right)-w\left(n_{t}^{*}\right)\right] \leqslant 0 .
$$

To this end, let $\left\{c_{t}, n_{t}, k_{t}\right\}_{t=0}^{\infty}$ be a feasible process. Fix $T \in \mathbb{N}_{+}$for the moment. Let

$$
\begin{aligned}
\Delta_{T} & =E \sum_{t=0}^{T} \beta^{t}\left[u\left(c_{t}\right)-w\left(n_{t}\right)\right]-E \sum_{t=0}^{T} \beta^{t}\left[u\left(c_{t}^{*}\right)-w\left(n_{t}^{*}\right)\right] \\
& \left.\leqslant E \sum_{t=0}^{T} \beta^{t}\left\{u^{\prime}\left(c_{t}^{*}\right)\left(c_{t}-c_{t}^{*}\right)-w^{\prime}\left(n_{t}^{*}\right)\left(n_{t}-n_{t}^{*}\right)\right)\right\}
\end{aligned}
$$


where $u^{\prime}\left(c_{t}^{*}\right)$ is the right derivative of $u$ at 0 if $c_{t}^{*}=0$, and similarly for $w^{\prime}\left(n_{t}^{*}\right)$. We have

$$
\begin{aligned}
\Delta_{T} \leqslant & E \sum_{t=0}^{T} \beta^{t}\left\{u ^ { \prime } ( c _ { t } ^ { * } ) \left[f\left(k_{t}, n_{t}, k_{t}^{*}, n_{t}^{*}\right)-f(t)\right.\right. \\
& \left.\left.\left.+\zeta\left(k_{t}-k_{t}^{*}\right)-\left(k_{t+1}-k_{t+1}^{*}\right)\right]-w^{\prime}\left(n_{t}^{*}\right)\left(n_{t}-n_{t}^{*}\right)\right)\right\} \\
\leqslant & E \sum_{t=0}^{T} \beta^{t}\left[u^{\prime}\left(c_{t}^{*}\right)\left(f_{1}(t)+\zeta\right)\left(k_{t}-k_{t}^{*}\right)\right. \\
& \left.+\left\{u^{\prime}\left(c_{t}^{*}\right) f_{2}(t)-w^{\prime}\left(n_{t}^{*}\right)\right\}\left(n_{t}-n_{t}^{*}\right)-u^{\prime}\left(c_{t}^{*}\right)\left(k_{t+1}-k_{t+1}^{*}\right)\right] .
\end{aligned}
$$

Recalling the first-order condition (3.4) for $n_{t}$, we see that for all $t \in \mathbb{Z}_{+}$,

$$
\left\{u^{\prime}\left(c_{t}^{*}\right) f_{2}(t)-w^{\prime}\left(n_{t}^{*}\right)\right\}\left(n_{t}-n_{t}^{*}\right) \leqslant 0 .
$$

Substituting into A.6 we obtain

$$
\begin{aligned}
\Delta_{T} \leqslant & E \sum_{t=0}^{T} \beta^{t}\left[u^{\prime}\left(c_{t}^{*}\right)\left(f_{1}(t)+\zeta\right)\left(k_{t}-k_{t}^{*}\right)-u^{\prime}\left(c_{t}^{*}\right)\left(k_{t+1}-k_{t+1}^{*}\right)\right] \\
=E & \sum_{t=0}^{T-1} \beta^{t}\left[-u^{\prime}\left(c_{t}^{*}\right)+\beta u^{\prime}\left(c_{t+1}^{*}\right)\left(f_{1}(t+1)+\zeta\right)\right]\left(k_{t+1}-k_{t+1}^{*}\right) \\
& \quad-\beta^{T} E u^{\prime}\left(c_{T}^{*}\right)\left(k_{T+1}-k_{T+1}^{*}\right) \\
=E & \sum_{t=0}^{T-1} \beta^{t}\left[-u^{\prime}\left(c_{t}^{*}\right)+\beta E_{t} u^{\prime}\left(c_{t+1}^{*}\right)\left(f_{1}(t+1)+\zeta\right)\right]\left(k_{t+1}-k_{t+1}^{*}\right) \\
& \quad-\beta^{T} E u^{\prime}\left(c_{T}^{*}\right)\left(k_{T+1}-k_{T+1}^{*}\right),
\end{aligned}
$$

where the last equality holds by the law of iterated expectations. Recalling the Euler condition (3.5) for $k_{t+1}$, we see that for all $t \in \mathbb{Z}_{+}$,

$$
\left[-u^{\prime}\left(c_{t}^{*}\right)+\beta E_{t} u^{\prime}\left(c_{t+1}^{*}\right)\left(f_{1}(t+1)+\zeta\right)\right]\left(k_{t+1}-k_{t+1}^{*}\right) \leqslant 0 .
$$

Substituting into A.12 we obtain

$$
\begin{aligned}
\Delta_{T} & \leqslant-\beta^{T} E u^{\prime}\left(c_{T}^{*}\right)\left(k_{T+1}-k_{T+1}^{*}\right) \\
& \leqslant \beta^{T} E u^{\prime}\left(c_{T}^{*}\right) k_{T+1}^{*} \rightarrow 0,
\end{aligned}
$$

where the second inequality holds since $k_{T+1} \geqslant 0$, and the convergence holds by the transversality condition (3.6). This completes the proof of Lemma 3.1 . 


\section{Appendix B Proof of Proposition 4.1}

Suppose that $\sigma=0$. Then conditions (4.5) and (4.6) can be written as

$$
\begin{aligned}
& s_{t}=1 \quad \Rightarrow \quad \rho \theta\left(k_{t}\right)^{\alpha+\bar{\alpha}}\left(n_{t}\right)^{\rho+\bar{\rho}-1}-\eta\left(n_{t}\right)^{\gamma} \begin{cases}=0 & \text { if } n_{t} \in(0,1), \\
0 & \text { if } n_{t}=1,\end{cases} \\
& s_{t}=0 \quad \Rightarrow \quad n_{t}=0 .
\end{aligned}
$$

The Euler condition for $k_{t+1}, 3.5$ can be written as

$$
\beta E_{t}\left[\alpha \theta\left(k_{t+1}\right)^{\alpha+\bar{\alpha}-1}\left(n_{t+1}\right)^{\rho+\bar{\rho}}+\zeta\right] \begin{cases}=1 & \text { if } k_{t+1} \in\left(0, g\left(k_{t}, n_{t}\right)\right), \\ \geqslant 1 & \text { if } k_{t+1}=g\left(k_{t}, n_{t}\right), \\ \leqslant 1 & \text { if } k_{t+1}=0 .\end{cases}
$$

The transversality condition 3.6 reduces to

$$
\lim _{T \rightarrow \infty} \beta^{T} E k_{T+1}=0 .
$$

Note that (B.1) and (B.2) can be combined into

$$
n_{t}=m\left(k_{t}, s_{t}\right) \equiv s_{t} \min \left\{\left[\frac{\rho \theta}{\eta}\left(k_{t}\right)^{\alpha+\bar{\alpha}}\right]^{\frac{1}{\gamma+1-\rho-\bar{\rho}}}, 1\right\} \text {. }
$$

Substituting into the left-hand side of $(\mathrm{B} .3)$ we have

$$
\begin{aligned}
& E_{t}\left[\alpha \theta\left(k_{t+1}\right)^{\alpha+\bar{\alpha}-1}\left(n_{t+1}\right)^{\rho+\bar{\rho}}+\zeta\right] \\
& =E_{t}\left[\alpha \theta\left(k_{t+1}\right)^{\alpha+\bar{\alpha}-1} m\left(k_{t+1}, s_{t+1}\right)^{\rho+\bar{\rho}}+\zeta\right] \\
& = \begin{cases}p_{01} h\left(k_{t+1}\right)+\zeta & \text { if } s_{t}=0, \\
p_{11} h\left(k_{t+1}\right)+\zeta & \text { if } s_{t}=1,\end{cases}
\end{aligned}
$$

where

$$
\begin{aligned}
h(k) & =\alpha \theta k^{\alpha+\bar{\alpha}-1} m(k, 1)^{\rho+\bar{\rho}} \\
& =\min \left\{\alpha \theta\left[\frac{\rho \theta}{\eta}\right]^{\frac{\rho+\bar{\rho}}{\gamma+1-\rho-\bar{\rho}}} k^{\frac{(\alpha+\bar{\alpha}-1)(\gamma+1)+\rho+\bar{\rho}}{\gamma+1-\rho-\bar{\rho}}}, \alpha \theta k^{\alpha+\bar{\alpha}-1}\right\} .
\end{aligned}
$$


Both expressions in the curly brackets are strictly decreasing in $k$ by 2.10 and (2.12). Thus $h(\cdot)$ is strictly decreasing, and the inverse $h^{-1}(\cdot)$ exists. Indeed, for $z>0$ we have

$$
h^{-1}(z)=\min \left\{\left[\frac{z}{\alpha \theta}\left[\frac{\eta}{\rho \theta}\right]^{\frac{\rho+\bar{\rho}}{\gamma+1-\rho-\bar{\rho}}}\right]^{\frac{\gamma+1-\rho-\bar{\rho}}{(\alpha+\bar{\alpha}-1)(\gamma+1)+\rho+\bar{\rho}}},\left[\frac{z}{\alpha \theta}\right]^{\frac{1}{\alpha+\bar{\alpha}-1}}\right\} .
$$

Note also that

$$
\lim _{k \downarrow 0} h(k)=\infty .
$$

Substituting (B.6) $-(\bar{B} .8)$ into $(\mathrm{B} .3)$ we have

$$
\beta\left[p_{s_{t} 1} h\left(k_{t+1}\right)+\zeta\right] \begin{cases}=1 & \text { if } k_{t+1} \in\left(0, g\left(k_{t}, n_{t}\right)\right), \\ \geqslant 1 & \text { if } k_{t+1}=g\left(k_{t}, n_{t}\right), \\ \leqslant 1 & \text { if } k_{t+1}=0\end{cases}
$$

where $p_{s_{t} 1}=p_{01}$ or $p_{11}$ depending on $s_{t}=0$ or 1 . For $p>0$ define

$$
q(p)=h^{-1}\left(\frac{1-\beta \zeta}{\beta p}\right) .
$$

Note from (B.12) that in $(\mathrm{B} .13)$, the case $k_{t+1}$ can be ruled out. Then (B.13) can be written as

$$
k_{t+1}=\min \left\{q\left(p_{s_{t} 1}\right), g\left(k_{t}, n_{t}\right)\right\} \text {. }
$$

We construct a process $\left\{c_{t}, n_{t}, k_{t}\right\}_{t=0}^{\infty}$ recursively as follows: given $k_{t}>0$ and $s_{t} \in\{0,1\}$, let

$$
n_{t}=m\left(k_{t}, s_{t}\right)
$$

Determine $k_{t+1}$ by $(\mathrm{B} .15)$. Let

$$
c_{t}=g\left(k_{t}, n_{t}\right)-k_{t+1} .
$$

Draw $s_{t+1}$ according to (4.1). Determine $n_{t+1}$ by (B.16), and so on. By construction, this process is feasible and satisfies (B.1)-(B.3). It also satisfies (B.4) by 2.13). Thus it is a sunspot equilibrium. The conclusion of the proposition now follows. 


\section{Proof of Proposition 4.2}

Suppose that $\bar{\alpha}=0$. Consider the stochastic optimal growth model 4.17)(4.19). The Euler condition for $k_{t+1}$ is written as

$$
\begin{aligned}
-u^{\prime}\left(c_{t}\right)+\beta E_{t} u^{\prime}\left(c_{t+1}\right)\left[s_{t+1} \alpha \theta\left(k_{t+1}\right)^{\alpha-1}\left(n_{t+1}\right)^{\rho+\bar{\rho}}+\zeta\right] & \begin{cases}=0 & \text { if } k_{t+1} \in\left(0, g\left(k_{t}, n_{t}\right)\right), \\
\geqslant & \text { if } k_{t+1}=g\left(k_{t}, n_{t}\right), \\
\leqslant & \text { if } k_{t+1}=0 .\end{cases}
\end{aligned}
$$

This is equivalent to the equilibrium Euler condition (3.5) for $k_{t+1}$ for the original economy 2.1$)-(2.3)$ with $\bar{\alpha}=0$ and $(4.3)$. The first-order condition for $n_{t}$ for the above stochastic optimal growth model is given by

$$
u^{\prime}\left(c_{t}\right) s_{t}(\rho+\bar{\rho}) \theta\left(k_{t}\right)^{\alpha}\left(n_{t}\right)^{\rho+\bar{\rho}-1}-\frac{\rho+\bar{\rho}}{\rho} w^{\prime}\left(n_{t}\right) \begin{cases}=0 & \text { if } n_{t} \in(0,1), \\ \geqslant 0 & \text { if } n_{t}=1, \\ \leqslant 0 & \text { if } n_{t}=0,\end{cases}
$$

which simplifies to

$$
u^{\prime}\left(c_{t}\right) s_{t} \rho \theta\left(k_{t}\right)^{\alpha}\left(n_{t}\right)^{\rho+\bar{\rho}-1}-w^{\prime}\left(n_{t}\right) \begin{cases}=0 & \text { if } n_{t} \in(0,1) \\ \geqslant 0 & \text { if } n_{t}=1 \\ \leqslant 0 & \text { if } n_{t}=0\end{cases}
$$

This is equivalent to 3.4 with $\bar{\alpha}=0$ and 4.3 . The transversality condition for the above problem is identical to (3.6).

Conditions (C.1) and (C.3) are necessary for optimality by standard arguments. The transversality condition $(3.6)$ is also necessary by the argument of Kamihigashi (2005, Section 6) $]^{4}$ Given that the sunspot variable $s_{t}$ is discrete, the existence of an optimal process for the optimal stochastic growth model (4.17)-(4.19) can easily be established by a standard argument (e.g., Ekeland and Sheinkman, 1986). Let $\left\{c_{t}, n_{t}, k_{t}\right\}_{t=0}^{\infty}$ be an optimal process for (4.17)-(4.19). Then by the above argument, it satisfies $(3.4)-(3.6)$. Thus by Lemma 3.1, it is an equilibrium of the original economy (2.1)-(2.3). Since it is a sunspot equilibrium, it follows that a sunspot equilibrium exists.

\footnotetext{
${ }^{4}$ The condition $\sigma \in[0,1]$ is needed here.
} 


\section{References}

Benhabib, J., Farmer, R.E.A., 1994, Indeterminacy and increasing returns, Journal of Economic Theory 63, 19-41.

Benhabib, J., Farmer, R.E.A., 1999, Chapter 6: Indeterminacy and sunspots in macroeconomics, Handbook of Macroeconomics, 1A, 387-448.

Benhabib, J., Meng, Q., Nishimura, K., 2000, Indeterminacy under constant returns to scale in multisector economies, Econometrica 68, 1541-1548.

Brock, W.A., Mirman, L.J., 1972, Optimal economic growth and uncertainty: the discounted case, Journal of Economic Theory 4, 479-513.

Clain-Chamosset-Yvrard, L., Kamihigashi, T., 2015, International transmission of bubble crashes: stationary sunspot equilibria in a two-country overlapping generations model, RIEB Discussion Paper DP2015-21, Kobe University.

Coury, T., Wen, Y., 2009, Global indeterminacy in locally determinate real business cycle models, International Journal of Economic Theory 5, 4960 .

Drugeon, J.-P., Wigniolle, B., 1996, Continuous-time sunspot equilibria and dynamics in a model of growth, Journal of Economic Growth 69, 24-52.

Drugeon, J.-P., Venditti, A., 2001, Intersectoral external effects, multiplicities \& indeterminacies, Journal of Economic Dynamics and Control 25, $765-787$.

Dufoourt, F., Nishimura, K., Vendittie, A., 2015, Indeterminacy and sunspots in two-sector RBC models with generalized no-income-effect preferences, Journal of Economic Theory 157, 1056-1080.

Ekeland, I., Scheinkman, S.A., 1986, Transversality conditions for some infinite horizon discrete time optimization problems, Mathematics of Operations Research 11, 216-229.

Farmer, R.E.A, Guo, J.-T., 1994, Real business cycles and the animal spirits hypothesis, Journal of Economic Theory 63, 42-72.

Grandmont, J.-M., 1989, Local bifurcation and stationary sunspots, Economic Complexity: Chaos, Sunspots, Bubbles, and Nonlinearity, 45-60.

Grandmont, J.-M., 1991, Expectations driven business cycles, European Economic Review 35, 293-299. 
Kamihigashi, T., 1996, Real business cycles and sunspot fluctuations are observationally equivalent, Journal of Monetary Economics 37, 105-117.

Kamihigashi, T., 1999, Chaotic dynamics in quasi-static systems: theory and applications, Journal of Mathematical Economics 31, 183-214.

Kamihigashi, T., 2002, Externalities and nonlinear discounting: indeterminacy, Journal of Economic Dynamics and Control 26, 141-169.

Kamihigashi, T., 2003, Necessity of transversality conditions for stochastic problems, Journal of Economic Theory 109, 140-149.

Kamihigashi, T., 2005, Necessity of the transversality condition for stochastic models with bounded or CRRA utility, Journal of Economic Dynamics and Control 29, 1313-1329.

Kamihigashi, T., 2008, Stochastic optimal growth with bounded or unbounded utility and with bounded or unbounded shocks, Journal of Mathematical Economics 43, 477-500.

Kamihigashi, T., 2015, Multiple interior steady states in the Ramsey model with elastic labor supply, International Journal of Economic Theory 11, $25-37$.

Mino, K., 2001, Indeterminacy and endogenous growth social constant returns, Journal of Economic Theory 97, 203-222.

Pelloni, A., Waldmann, R., 1998, Stability properties of a growth model, Economics Letters 61, 55-60.

Pintus, P.A., 2006, Indeterminacy with almost constant returns to scale: capitallabor substitution matters, Economic Theory 28, 633-649.

Puterman, M.L., 2005, Markov Decision Processes: Discrete Stationary Dynamic Programming, Hoboken, NJ: John Wiley \& Sons. 